\title{
Unsolved problems of noise
}

\author{
Peter V. E. McClintock
}

Random noise, and its effect on physical systems, has always been an interdisciplinary subject. Using the study of fluctuations as a unifying theme can lead to interesting scientific conferences, such as the recent UPoN'99 meeting in Adelaide, Australia*

Over the years, noise has attracted some of the best scientists: in 1828 the botanist Robert Brown ${ }^{1}$ discovered the random fluctuations of tiny particles in a fluid, which remained a mystery until Einstein's epochmaking paper ${ }^{2}$ on Brownian motion in 1905. But it was not until later that the ubiquitous presence of noise began to be appreciated. A milestone was Johnson's 1927 paper $^{3}$ in which he identified noise in electrical circuits as being of thermal origin. In the middle of the twentieth century, noise seemed merely a nuisance - to be eliminated or minimized wherever possible. It spoiled radio reception and musical recordings, and limited the precision of physical measurements. Later, it was found to wash out the fine structure of beautiful fractal patterns generated by nonlinear systems.

But noise is much more than just a nuisance. By the 1970s it became apparent that noise can play a creative role too. One of the first examples was the observation of noise-induced transitions ${ }^{4}$, whereby the state of a nonlinear system can be utterly changed by the introduction of noise above a critical intensity. Another was stochastic resonance, in which a weak periodic signal in a nonlinear system can be amplified by added noise. Stochastic resonance was introduced $^{5,6}$ to try to explain the Earth's ice-age cycle, but it is now recognized to be far more common, occurring for example in lasers, electronic circuits and sensory neurons. Yet another creative effect of noise is seen in Brownian ratchets7 where a net current of particles can be driven by noise, providing that there is an appropriate asymmetry in the system: this particular phenomenon may underlie the transport of macromolecules within biological cells. And, of course, it is noise in the sense of thermal fluctuations that drives chemical reactions, including those responsible for life itself.

The negative aspects of noise are still with us and, given its Janus-like character, how is noise to be perceived, pursued and investigated? A proper appreciation of noise must involve studies of both the mechanisms that produce it, for example in semiconductor devices, and the effect that it has on systems subject to it. Both these aspects were well represented at UPoN'99.

Of the several kinds of Brownian ratchet that have been considered, the "flashing ratchet" has attracted particular attention because of its likely relevance to biology ${ }^{7}$. In this ratchet, a noise-driven particle which might be attached to a vesicle carrying a macromolecule - diffuses within a periodic potential (a cyclic variation of the particles potential energy with position). This potential can typically take one of two forms - it can either be flat, with no variation at all, or it can have a series of ratchet-shaped teeth. The system flashes back and forth between these two configurations. As a result - depending on the shape of the teeth and the characteristics of the noise - the particle will diffuse preferentially either to the right or to the left; given noise of appropriate intensity, there will be a net current. Remarkably, there is still a net current of particles, albeit a smaller one, even if the potential is tilted so that the particles have to run uphill. Yet if the flashing ceases, the system stops working. Current will not flow uphill for either of the two configurations taken alone.

Surprising though it may seem, there appears to be a close relationship between flashing ratchets and certain mathematical games. Juan Parrondo (Universidad Complutense de Madrid, Spain) has devised two simple games whose behaviour was shown by Derek Abbott (University of Adelaide and organizer of UPoN'99) and colleagues to model closely that of a flashing ratchet. Either game played on its own results in loss for the player. But if play alternates randomly between the two games the result is a win - that is, two losing strategies can result in a win.

* Second International Conference on Unsolved Problems of Noise and Fluctuations (UPoN'99), Adelaide, Australia, 1115 July 1999. Proceedings to be published by the American Institute of Physics. 
The games are amenable to rigorous mathematical analysis, and promise to pave the way to a detailed understanding of ratchet phenomena. Such models could have a bearing on population genetics and evolutionary biology, as well as on game theory in economics.

In surveying stochastic resonance, Sergey Bezrukov (National Institutes of Health, Bethesda, Maryland) admitted ruefully that early perceptions now seem inadequate. In particular, the once standard definition ${ }^{8}$ of stochastic resonance as an effect confined to bistable systems that can exist in two different states (including systems with thresholds) is not very useful because the phenomenon occurs much more widely. In fact, the only general definition of stochastic resonance that remains tenable is that the effect arises whenever a nonlinear system has a large, strongly noise-dependent susceptibility ${ }^{9}$ (a quantity that relates the periodic response of the system to the weak periodic force that gives rise to it).

What do stochastic resonance, Brownian ratchets and, indeed, most other fluctuational phenomena have in common? It has slowly been realized that a unifying feature in many cases is reliance on "special" fluctuations that occur infrequently. For example, a system will usually wait for a long time below a potential barrier for a fluctuation of exactly the right shape and strength to carry it over. These large, rare fluctuations are of fundamental importance in understanding the behaviour of noise-driven systems. They are starting to be understood, even in the dauntingly complex, non-equilibrium cases found in practical situations.

Advances have recently been made in experimenting on large, rare fluctuations. Riccardo Mannella (University of Pisa, Italy) described experiments that have confirmed the validity of the so-called logarithmic susceptibility, a new physical quantity ${ }^{10}$ for describing large fluctuations in periodically driven non-equilibrium systems. These results are immensely encouraging because, if large fluctuations can be properly understood, it may then be possible to control their destructive power (for example, in the spontaneous failure of lasers) and, perhaps, to exploit them (such as when minimizing the energy requirements for desired transitions). Noise is starting to look altogether friendlier and more useful than it did in earlier times.

Peter V. E. McClintock is in the Department of Physics, University of Lancaster, Lancaster LA1 $4 \mathrm{YB}, \mathrm{UK}$.

e-mail: p.v.e.mcclintock@lancaster.ac.uk

\section{References}

[1] Brown, R. Phil. Mag. 4, 161 (1828).

[2] Einstein, A. Ann. Phys. 17, 549 (1905).

[3] Johnson, J. B. Nature 119, 50-51 (1927).

[4] Horsthemke, W. \& Lefever, R. Noise-Induced Transitions (Springer, Berlin, 1984).

[5] Benzi, R., Sutera, S. \& Vulpiani, A. J. Phys. A 14, L453L457 (1981).

[6] Nicolis, C. Tellus 34, 19 (1982).

[7] Bier, M. Contemp. Phys. 38, 371-379 (1997).

[8] Gammaitoni, L., Hnggi, P., Jung, P. H. \& Marchesnoi, F. Rev. Mod. Phys. 70, 223-288 (1998).

[9] Dykman, M. I., Mannella, R., McClintock, P. V. E. \& Stocks, N. G. Phys. Rev. Lett. 65, 2606 (1990).

[10] Smelyanskiy, V. N., Dykman, M. I., Rabitz, H. \& Vugmeister, B. E. Phys. Rev. Lett. 79, 3113-3116 (1997). 